\title{
Obtenção de vidros porosos para uso como dispositivo de liberação de feromônios
}

\section{(Porous glass processing for use as pheromones release device)}

\author{
R.M. Takahashi, O.B.G.Assis \\ Embrapa Instrumentação Agropecuária, Rua XV de Novembro 1452, C.P. 741, S. Carlos, SP 13560-970 \\ odílio@cnpdia.embrapa.br
}

\begin{abstract}
Resumo
Peças sinterizadas porosas para emprego como matrizes de liberação controlada de feromônios foram produzidas a partir de pós de vidro de origem comercial. As peças, no formato cilíndrico, foram sinterizadas a $700{ }^{\circ} \mathrm{C}$ e apresentam tamanho médio de poros da ordem de 35,80 $\mu \mathrm{m}$ e porosidade aparente de 37,45\%. O feromônio sintético (E)-2-hexenal foi admitido na matriz por ação de capilaridade e ensaios de permeabilidade e liberação realizados em condições ambientais não controladas. Dispositivo similar comercial (lure polimérico) nas mesmas dimensões foi avaliado em caráter comparativo. As peças sinterizadas apresentaram uma menor permeabilidade e taxa de absorção do líquido inferior ao similar comercial, mas com uma maior taxa de liberação (evaporação) do composto volátil. Embora estruturalmente diferente da peça polimérica comercial, os elementos sinterizados podem satisfatoriamente substituir as importadas, com eficiência similar.
\end{abstract}

Palavras-chave: vidro sinterizado, processamento de elementos porosos, liberação controlada, feromônios.

\begin{abstract}
Porous sintered glasses for use as pheromone controlled deliver matrix were produced from glass powder. The pieces, in cylindrical form, were sintered at $700^{\circ} \mathrm{C}$ resulting in average porous size of $35.80 \mu \mathrm{m}$ and apparent porosity of $37.45 \%$. Synthetic pheromone (E)2-hexenal was uptake into the matrix by capillarity forces and permeability and deliver tests carried out at ordinary environmental conditions. Similar commercial device (polymeric lure) in the same size and dimensions was also tested for comparative data. The sintered piece presented inferior permeability and liquid absorption rate, though with higher deliver rate (evaporation) of the volatile compound. Although structurally different from the commercial polymeric piece, the sintered elements can satisfactory replace the imported devices with similar efficiency.
\end{abstract}

Keywords: sintered glass, porous elements processing, controlled deliver, pheromones.

\section{INTRODUÇÃO}

Feromônios consistem em uma classe de compostos denominados semioquímicos, ou seja, são substâncias produzidas por um organismo que induz uma resposta em um outro organismo. Vários seres vivos liberam feromônios, mas principalmente os insetos têm por base a comunicação através de estímulos semioquímicos. A detecção desses compostos voláteis ocorre por receptores biológicos que interagem e identificam a mensagem mesmo em concentrações extremamente baixas e em distâncias relativamente longas [1]. Feromônios sintéticos têm sido amplamente empregados no monitoramento e captura de insetos para controle de pestes na lavoura [2], por meio da liberação controlada fazendo uso de elementos porosos, conhecidos como "lures", nos quais os compostos são embebidos e adequadamente acomodados em armadilhas ou em recipientes de liberação unidirecional. Os "lures" comerciais consistem em pequenas peças de formatos diversos, na sua maioria confeccionadas de polímeros, em matrizes porosas de alta área superficial, nas quais os compostos são admitidos por capilaridade. A maioria desses dispositivos é importada, sem similares nacionais, sendo que a liberação se dá por evaporação pelas diversas faces das peças e o elemento é descartado após o uso.

Neste trabalho, elementos vítreos porosos são confeccionados por sinterização e caracterizados com respeito à porosidade, permeabilidade, absorção e liberação de feromônio sexual sintético, comparando os resultados com peça polimérica de formato similar de origem comercial.

\section{PROCEDIMENTO EXPERIMENTAL}

\section{Materiais}

A matéria prima inicial constituiu de laminas de vidro plano na composição química média de $\mathrm{SiO}_{2}(72,8 \%), \mathrm{Na}_{2} \mathrm{O}$ $(13,2 \%), \mathrm{CaO}(11,2 \%), \mathrm{MgO}(0,16 \%), \mathrm{Al}_{2} \mathrm{O}_{3}(2,13 \%), \mathrm{FeO} /$ $\mathrm{Fe}_{2} \mathrm{O}_{3}(0,039 \%), \mathrm{K}_{2} \mathrm{O}(0,09 \%)$, segundo determinação da Saint-Gobain (Centro Técnico de Elaboração do Vidro). Este 
material foi escolhido por se tratar de material facilmente encontrado no comércio. As laminas foram fragmentadas manualmente e os pós foram obtidos após moagem em moinho de bolas de alumina, em rotação de $36 \mathrm{rpm}$, para cargas individuais de $200 \mathrm{~g}$. Após $2 \mathrm{~h}$ de cominuição, os pós foram classificados por peneiramento e separados em tamanhos de partículas da ordem de $50 \mu \mathrm{m}$. Não foram realizadas análises da composição após a moagem.

A prensagem se deu em matriz metálica, no formato cilíndrico, nas dimensões de $5 \mathrm{~mm}$ altura por $12 \mathrm{~mm}$ de diâmetro, para pressão uniaxial não superior a $100 \mathrm{MPa}$. As pastilhas foram processadas com adição de $10 \%$ em massa de ácido oléico (densidade $0,891 \mathrm{~g} / \mathrm{mL}$ ), com a finalidade de proporcionar resistência mecânica a verde dos corpos. A sinterização foi realizada ao ar em uma mufla com taxa de aquecimento de $10{ }^{\circ} \mathrm{C}$ por minuto com patamar de sinterização de $700{ }^{\circ} \mathrm{C}$ por $1 \mathrm{~h}$. O resfriamento se deu dentro do forno até temperatura ambiente para evitar o surgimento de trincas por choque térmico.

\section{Caracterização}

A caracterização das peças sinterizadas seguiu procedimentos padrões de porosimetria por intrusão de mercúrio (Pore Sizer 9320, Micrometrics) e de medidas de permeabilidade em sistema de queda de pressão em câmara cilíndrica com fluxo perpendicular à amostra, conforme princípio ilustrado na Fig. 1 e detalhadamente descrito em [3]. O diâmetro de trabalho foi de $10 \mathrm{~mm}$, o que significa uma área de penetração de fluxo na amostra próxima a $78,5 \mathrm{~mm}^{2}$. A queda de pressão foi estabelecida por diferença medida em sensor eletrônico, para passagem de ar comprimido para pressões iniciais de 0,5 a $3,5 \mathrm{MPa}$, considerando-se a densidade do ar $\rho=1,079 \mathrm{~kg} / \mathrm{m}^{3}$ e a viscosidade $\mu=1,83 \mathrm{x}$ $10^{-5}$ Pa.s.

As medidas foram realizadas na temperatura ambiente e em duplicatas para garantir repetitividade. Os dados foram calculados tendo por base os conceitos de permeabilidade

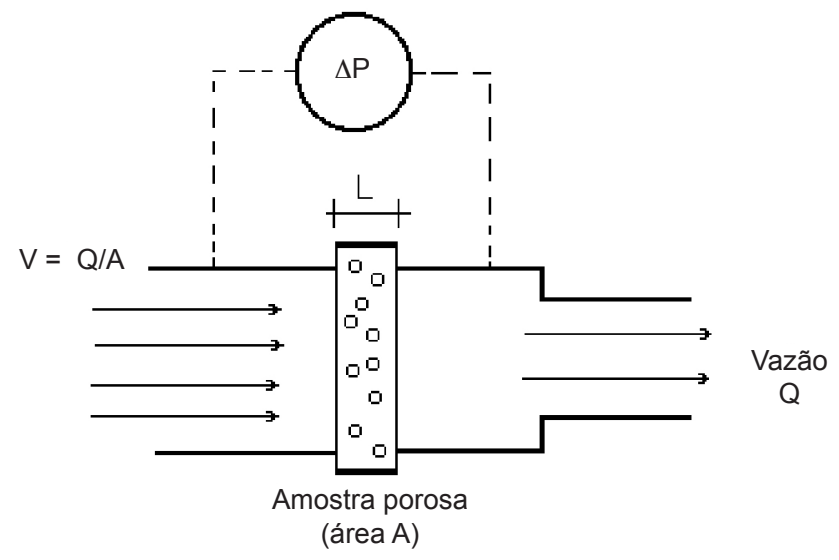

Figura 1: Princípio de medida para o sistema de permeação por queda de pressão.

[Figure 1: Scheme of the permeation system by pressure-drop.]
Darciana [4].

A porosidade aparente foi quantificada pelo método de Arquimedes, pela relação:

$$
P A(\%)=\left[\left(\mathrm{M}_{u}-\mathrm{M}_{s}\right) /\left(\mathrm{M}_{u}-\mathrm{M}_{i}\right)\right] \times 100
$$

sendo $\mathrm{M}_{\mathrm{s}}$ a massa seca, $\mathrm{M}_{\mathrm{i}}$ a massa do corpo imerso em água e $\mathrm{M}_{\mathrm{u}}$ a massa úmida (após imersão em água).

\section{Liberação de feromônio}

O feromônio empregado foi (E)-2-hexenal de fórmula $\mathrm{C}_{6} \mathrm{H}_{10} \mathrm{O}$ (cedido pela Embrapa Recursos Genéticos CENARGEN) de densidade $1,13 \mathrm{mg} / \mathrm{mL}$. A admissão na matriz porosa se deu por imersão total das peças no composto seguido de ação de vácuo em dessecador para a total eliminação de gases internos e conseqüente preenchimento dos poros. A fração absorvida foi estimada segundo a relação:

$$
A(\%)=\left[\left(\mathrm{M}_{\mathrm{u}}-\mathrm{M}_{s}\right) / \mathrm{M}_{s}\right] \times 100
$$

A taxa de liberação foi monitorada em tempo real por medida em balança analítica (Gehaka, AG 200) conectada a um computador por um cabo serial. Foram registradas automaticamente noventa medidas por minuto até $210 \mathrm{~min}$, na temperatura ambiente de $26 \pm 2{ }^{\circ} \mathrm{C}$ e umidade relativa $76 \%$. A título de comparação, todos os ensaios foram também conduzidos nas peças comerciais, lure plástico da New Serrico (Fuji Flavor Co., Ltd., Japão).

\section{RESULTADOS E DISCUSSÃO}

O processo de sinterização de vidros sódio-cálcicos é bem conhecido e ocorre como conseqüência de fluxo viscoso para temperaturas entre 600 e $800{ }^{\circ} \mathrm{C}$, segundo mecanismos já detalhados [5]. A Fig. 2 mostra os gráficos gerados pelas análises de porosimetria de mercúrio nos quais o incremento de volume em função do diâmetro de poros indica que a pastilha comercial apresenta uma faixa mais extensa de distribuição de tamanho de poros e com intensidade em seu diâmetro médio maior que a peça processada por sinterização. A grande maioria é de poros abertos para ambos os materiais sendo que os diâmetros médios medidos são bastante próximos, ou seja, de 42,19 $\mu \mathrm{m}$ para o lure comercial e de $35,80 \mu \mathrm{m}$ para o vidro sinterizado.

O estabelecimento de uma estrutura de poros interconectados em maior ou menor dimensão e em variação volumétrica interfere no escoamento e admissão do fluído alterando as características de liberação [6]. Uma maior tortuosidade, associada a uma menor porosidade geram redução na permeabilidade total. Essa característica pode ser inferida através das curvas de queda de pressão, expressas na Fig. 3. O parâmetro de pressão é definido como a razão entre a pressão medida antes e após a passagem pelo meio poroso, segundo a relação: 


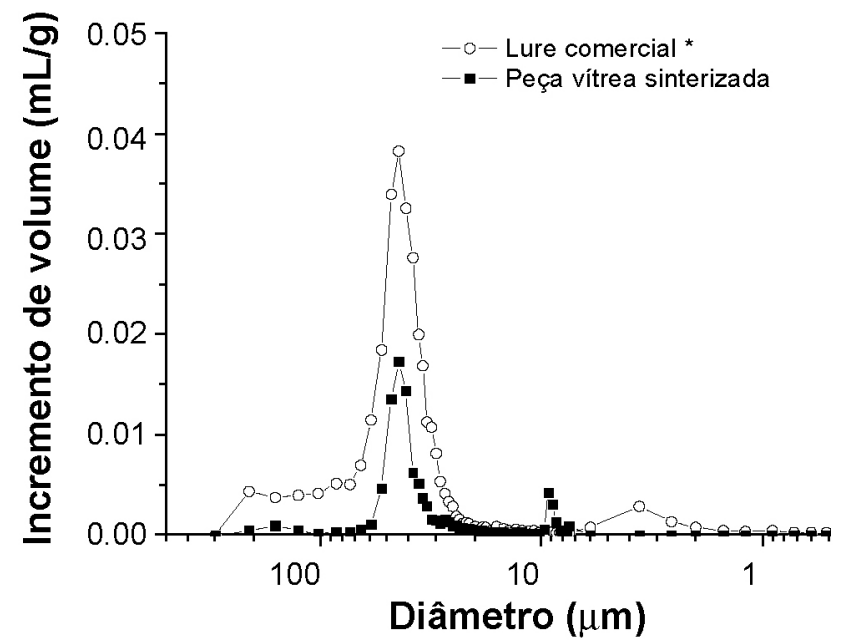

Figura 2: Variação de volume com do diâmetro de poros na amostra cerâmica e de referência (*elemento plástico da New Serrico, Fuji Flavor Co., Ltd., Japão).

[Figure 2: Volume variation as a function of porous size in the ceramic and the reference samples (*plastic piece from New Serrico, Fuji Flavor Do., Ltd., Japan).]

$$
\frac{\mathrm{P}_{\mathrm{f}}^{2-} \mathrm{P}_{0}^{2}}{2 \times \mathrm{P}_{\mathrm{atm}} \times \mathrm{L}}
$$

sendo L a espessura da peça, $\mathrm{P}_{\mathrm{atm}}$ a pressão atmosférica local e $\mathrm{P}_{0}$ e $\mathrm{P}_{\mathrm{f}}$ respectivamente a pressão anterior e posterior a passagem pela membrana. Menores valores deste parâmetro indicam uma maior permeabilidade e vice-versa [7], ou seja, menores parâmetros de pressão em função da velocidade superficial do fluído indicam uma maior facilidade de liberação do líquido presente nos poros. Os resultados

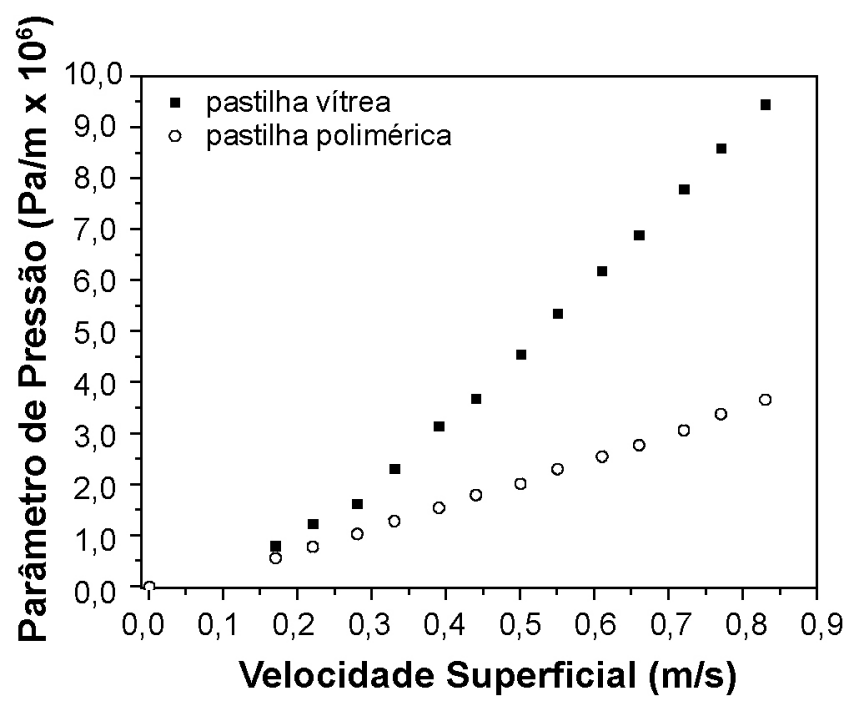

Figura 3: Curvas experimentais da queda de pressão através das amostras comerciais (pastilha polimérica) e vítrea sinterizada.

[Figure 3: Pressure-drop experimental curves through commercial (plastic piece) and vitreous sintered samples.]

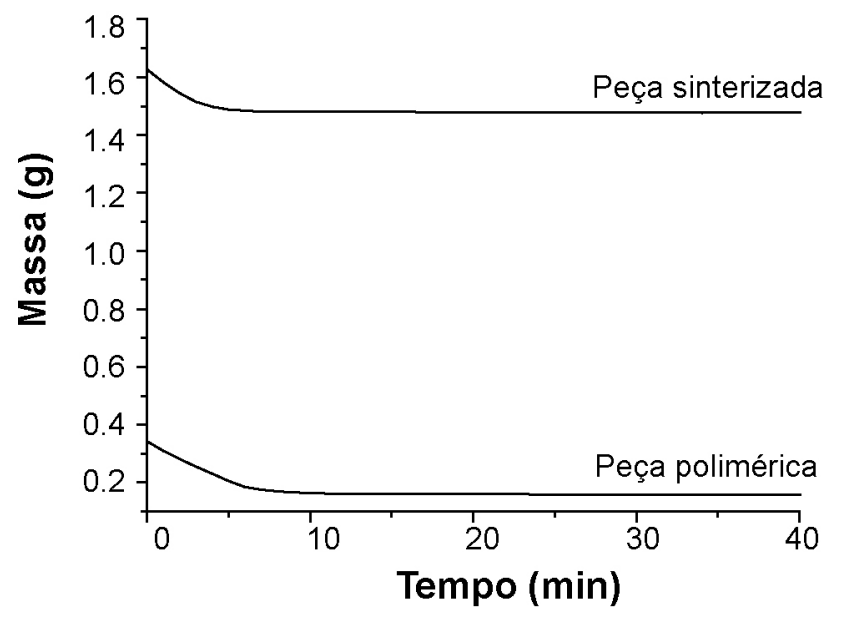

Figura 4: Perda de massa em função do tempo nas amostras com o feromônio $\mathrm{C}_{6} \mathrm{H}_{10} \mathrm{O}$, a $27 \pm 2^{\circ} \mathrm{C}$ e UR $76 \%$.

[Figure 4: Mass loss as a function of time in samples soaked with pheromone $\mathrm{C}_{6} \mathrm{H}_{10} \mathrm{O}$, at $27 \pm 2{ }^{\circ} \mathrm{C}$ and $76 \% \mathrm{RH}$ gradient.]

obtidos indicam que o elemento polimérico apresenta uma alta permeabilidade comparada com a peça sinterizada, o que deve resultar, em princípio, em uma maior taxa de absorção e de liberação de feromônio.

Esses dados são corroborados pela medida de porosidade aparente que indica vazios totais da ordem de $50,40 \%$ na peça polimérica e de $37,45 \%$ na peça sinterizada. A medida de absorção para as peças poliméricas é consideravelmente superior à das vítreas: as pastilhas sinterizadas absorvem no máximo de $26,18 \%$ de seu peso e o lure polimérico impregna em sua estrutura o dobro, ou seja, absorve o correspondente $104,3 \%$ de seu peso inicial em feromônio. Os ensaios realizados por perda de massa em tempo real, contudo, indicam perfis bastante próximos com relação a volatização em ambas as peças, diferenciando apenas com respeito a massa matricial inicial de cada elemento (Fig. 4). Este aspecto torna-se distinto quando observado os valores das derivadas, e conseqüente, o cálculo da taxa de liberação (Fig. 5).

Embora a matriz polimérica apresente porosidade e permeabilidade maiores que a similar vítrea e tenha uma liberação volumétrica inicial maior (valor inicial no eixo das ordenadas, Fig. 5), o perfil das derivadas indica uma menor taxa de volatilização do feromônio, ou seja, calculando a partir da Fig. 4, temos $(\Delta \mathrm{M} / \Delta \mathrm{t})_{\mathrm{pol}}=6,83 \times 10^{-3} \mathrm{~g} \cdot \mathrm{min}^{-1} \mathrm{e}$ $(\Delta \mathrm{M} / \Delta \mathrm{t})_{\text {vidro }}=8,14 \times 10^{-3} \mathrm{~g} \cdot \mathrm{min}^{-1}$. Esses resultados, embora de mesma ordem de grandeza, são indicativos de que assim como há uma maior absorção há igualmente uma maior retenção ou afinidade do feromônio na matriz polimérica do que na vítrea, o que pode ser entendido em termos de interações hidrofóbicas/hidrofílicas ou de capilaridade, considerando que ambos os materiais avaliados diferem nessas características.

De um modo geral as peças sinterizadas apresentam características bastante similares ao sistema importado, 


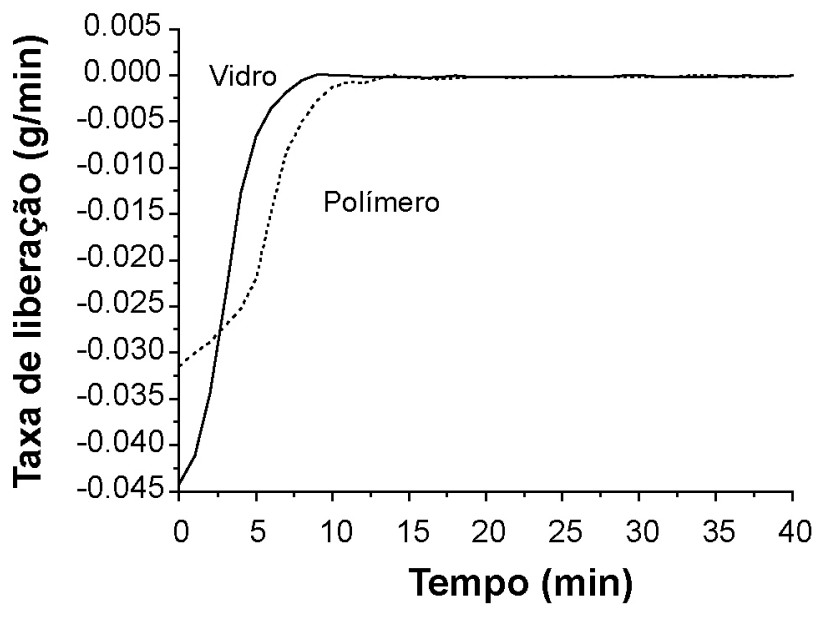

Figura 5: Curvas das derivadas da perda de massa, indicando taxa de volatilização de $6,83 \times 10^{-3} \mathrm{~g} \cdot \mathrm{min}^{-1}$ para o lure e de $8,14 \times 10^{-3}$ g. $\min ^{-1}$ para a matriz sinterizada.

[Figure 5: Derivative curves of mass loss, resulting in a volatilization rate of $6.83 \times 10^{-3} \mathrm{gmin}^{-1}$ for the plastic lure and $8.14 \times 10^{-3} \mathrm{gmin}^{-1}$ for the sintered matrix.]

podendo vir a ser uma alternativa para suprir o mercado nacional no seguimento de dispositivos liberadores de voláteis para uso agroindustrial.

\section{CONCLUSÕES}

Peças vítreas porosas foram processadas e apresentam poros abertos e capacidade de retenção de feromônio em sua matriz por ação de capilaridade. A porosidade e retenção foram inferiores a do dispositivo polimérico comercial, mas com taxas de liberação (volatilização) bastante similares. Embora estruturalmente diferentes das peças poliméricas comerciais, os elementos sinterizados podem satisfatoriamente substituir as importadas, com eficiência satisfatória.

\section{AGRADECIMENTOS}

À Embrapa pelo suporte financeiro.

\section{REFERÊNCIAS}

[1] W. Olkowski, S. Daar, H. Olkowski, The Gardener's guide to Common-Sense Pest Control, Taunton, New Ed edition (1995) 320p.

[2] L. Ryan, Post-Harvest Tobacco Infestation Control. Springer, $1^{\text {st }}$ Ed. (1995) 168p.

[3] R. A. Vasques, M. D. M. Innocentini, O. B. G. Assis, Rev. Fís. Aplic. Instrum. 14, 4 (1999) 120-123.

[4] M. D. M. Innocentini, V. R. Salvini, V. C. Pandolfelli, Am.Ceram. Soc. Bull. 77, 11 (1999) 64-68.

[5] G. W. Scherer. J. Am. Ceram. Soc. 60, 5-6 (1976) 236239.

[6] S. B. Iversen, V.K. Bhatia, K. Dam-Johansen, G. Jonsson, J. Membrane Sci. 130, 1-2 (1997) 205-217.

[7] M. D. M. Innocentini ,V. R. Salvini, J. R. Coury, V. C. Pandolfelli, "The Permeability of Ceramic Foams", Am. Ceram. Soc. Bull. 78, 9 (1999) 78-84.

(Rec. 20/02/2008, Rev. 23/05/2008, Ac. 20/06/2008) 